Revista de Psicología Vol. 35 (2), 2017 (ISSN 0254-9247)

\title{
Validez de contenido y confiabilidad inter-observadores de Escala Integral Calidad de Vida
}

\author{
Alicia Boluarte Carbajal ${ }^{1}$ y Kotaro Tamari ${ }^{2}$ \\ Instituto Nacional de Rehabilitación "Dra. Adriana Rebaza Flores" \\ Amistad Perú-Japón
}

\begin{abstract}
Se evaluaron las propiedades psicométricas de la Escala Integral de Calidad de Vida: Versión Objetiva. Se obtuvo evidencias de validez por contenido mediante el coeficiente V de Aiken con participación de 10 jueces expertos y análisis de fiabilidad inter-observador en una muestra de 45 personas pertenecientes a 15 familias con presencia de una persona con discapacidad intelectual. Los resultados muestran una insuficiente evidencia de validez de jueces en cuanto a los criterios de claridad, coherencia, relevancia y suficiencia presentados. La fiabilidad inter-observador reporta un coeficiente Krippendorff's Alpha total de .58, correspondiente a nivel moderado. Se obtuvo una nueva versión de la Escala Integral de Calidad de Vida: Versión Objetiva para personas con discapacidad intelectual adaptada a las características de la realidad sociocultural de Lima, Perú.

Palabras claves: Calidad de Vida, discapacidad intelectual, validez de contenido, confiabilidad, rehabilitación.
\end{abstract}

Content validity and inter-rater reliability of the Comprehensive Quality of Life Scale The aim of this study was to assess the psychometric properties of the Comprehensive Quality of Life Scale, Objective Version. Content validity was obtained using Aiken's V coefficient with the participation of 10 experts and inter-rater reliability was assessed with a sample of 45 participants from 15 families with presence of an intellectual disabled. The results showed insufficient validity in the criteria of clarity, consistency and relevance. The inter-observer reliability was moderate with an overall coefficient of .58. A new version of the scale was obtained for intellectual disabled persons, adapted to the sociocultural characteristics of Lima, Perú.

1 Magister en Psicología por la Universidad Nacional Mayor de San Marcos y Jefa de la Unidad Funcional de Investigación y Desarrollo de Tecnología del Instituto Nacional de Rehabilitación. Av. Defensores del Morro 264, Lima 09, Perú. Contacto:aliciaboluarte@gmail.com

$2 \mathrm{PhD}$ por Curtin Universitiy, Western Australia. Voluntario de la Agencia de Cooperación Internacional del Japón. Edificio Torre Siglo XXI, Piso 21, Av. Canaval Moreyra 380, Lima 27, Perú. Contacto: ktamari@hotmail.com 
Keywords: Integral Quality of Life Scale, intellectual disability, content validity, reliability, rehabilitation.

\section{Validade de conteúdo e fiabilidade inter-observadores de Escala Integral Qualidade de} Vida

Avaliaram-se as propriedades psicométricas da Escala Integral de Qualidade de Vida: Versão Objetiva. Obteve-se evidências de validade por conteúdo mediante o coeficiente V de Aiken com participação de 10 juízes experientes e análises de confiabilidade inter-observador em uma mostra de 45 pessoas pertencentes a 15 famílias com presença de uma pessoa com discapacidade intelectual. Os resultados mostram uma insuficiente evidência de validade de juízes em quanto aos critérios de clareza, coerência, relevância e suficiencia apresentados. A confiabilidade inter-observador reporta um coeficiente Krippendorff's Alpha total de .58, correspondente a nível moderado. Obteve-se uma nova versão da Escala Integral de Qualidade de Vida: Versão Objetiva para pessoas com discapacidade intelectual adaptada às características da realidade sociocultural de Lima, Peru.

Palavras finque: Qualidade de Vida, discapacidade intelectual, validade de conteúdo, fiabilidade, reabilitação. 
La calidad de vida en personas con discapacidad intelectual se orienta dentro de la línea de investigación de la psicología positiva, definida por Seligman et al. (1999) como el estudio científico de las experiencias positivas, los rasgos individuales positivos, las instituciones que facilitan su desarrollo y los programas que ayudan a mejorar la calidad de vida de los individuos, mientras previene o reduce la incidencia de la psicopatología. Dicho estudio mantiene una relevancia teórica y práctica debido a que contribuye con un proceso de transformación positiva y significativa de mejores prácticas profesionales, organizativas y de una política social (Verdugo, 2001).

El concepto de calidad de vida representa "Un término multidimensional de las políticas sociales que significa tener buenas condiciones de vida 'objetivas' y un alto grado de bienestar 'subjetivo', y también incluye la satisfacción colectiva de necesidades a través de políticas sociales en adición a la satisfacción individual de necesidades...” (Palomba, 2002, p. 3).

La calidad de vida es un constructo teórico que en los últimos años ha cobrado especial relevancia, convirtiéndose en un principio básico de prestación de servicios, con un modelo ecológico bajo la perspectiva de tres sistemas: el microsistema, referido al entorno social inmediato, la familia, el trabajo; el mesosistema, que incluye el vecindario, la comunidad, las instituciones de servicios; y el macrosistema, relacionado con las tendencias sociopolíticas, económicas y con los factores sociales (Gómez, Verdugo \& Arias, 2010). Estos tres sistemas son interdependientes e influyen en la estructura de valores y creencias de las personas. Las investigaciones sobre calidad de vida en personas con discapacidad intelectual se orientan a promover una política social centrada en la persona y en una mejor planificación de los apoyos, ya que la discapacidad en general —y, específicamente, la intelectual — está asociada a situaciones de marginación y exclusión social, lo que pone en riesgo su calidad de vida y la de sus familias (Giné, 2004). 
Las personas con discapacidad intelectual presentan problemas en su desenvolvimiento personal-social que les impiden adaptarse y responder a las exigencias y expectativas sociales, siendo excluidos de los ámbitos formales de la educación y trabajo. Esta situación las convierte, de acuerdo a Córdoba, Mora, Bedoya y Verdugo (2007), en un grupo de alta vulnerabilidad por las dificultades en la generación y mantenimiento de redes de apoyo. Una revisión reciente de la literatura especializada sustenta esta afirmación, al mostrar que las personas con discapacidad intelectual participan menos en actividades recreativas, lo que a su vez se relaciona con una disminución de sus vínculos sociales y conlleva a la exclusión social (Merrels, Buchanan \& Waters, 2017). Esto es confirmado por Boluarte, Méndez y Martell (2006), quienes indican que la carencia de estimulación psicosocial genera una disminución y deterioro funcional progresivo en la persona con déficit intelectual, incrementándose la presencia de conductas psicopatológicas.

En la actualidad, la perspectiva de trabajo hacia la inclusión socio laboral de las personas con discapacidad intelectual difiere de las empleadas tradicionales en que no solo basta con garantizar la salud y seguridad de las personas, sino que se debe orientar hacia la calidad de vida y autodeterminación de cada persona (Verdugo, Sainz \& Gómez, 2009). Por ejemplo, Curryer, Stancliffe y Dew (2015) señalan la necesidad de fomentar la autodeterminación en las personas con discapacidad intelectual, es decir, que tengan la posibilidad de tomar decisiones relevantes en sus vidas, con un grado mínimo de coerción por parte de factores externos. De hecho, la evidencia sugiere que, cuando se toma en cuenta los deseos y metas personales de las personas con discapacidad intelectual, se obtienen mejores resultados relacionados con una mayor calidad de vida (Lombardi, Croce, Claes, Valdevelde \& Schalock, 2016).

Dicho énfasis se ha visto reflejado en la adopción de criterios funcionales para evaluar la discapacidad intelectual. Por ejemplo, la Asociación Americana de Psiquiatría (2014) ha realizado recientemente cambios importantes en los criterios diagnósticos para la discapacidad intelectual, la cual ya no es determinada únicamente por los puntajes de cociente intelectual, sino que se considera el funcionamiento integral de 
la persona en relación con su entorno social. Esto se aprecia con mayor claridad en los criterios propuestos por la Asociación Americana de Discapacidades Intelectuales y del Desarrollo, los cuales resaltan el nivel de apoyo que las personas con discapacidad intelectual requieren, más que en su habilidad personal desligada de su entorno social (Thompson et al., 2009). Como señalan Barlow y Durand (2015), la definición de discapacidad intelectual es establecida socialmente y, por lo tanto, puede variar de acuerdo con los cambios en la propia sociedad; así pues, la discapacidad intelectual no constituye una característica intrínseca a la persona que la padece, sino que depende en última instancia de la forma en que esta se relaciona con el contexto social que le tocó vivir.

La nueva concepción de la discapacidad intelectual incluye los constructos de inteligencia, conducta adaptativa y calidad de vida (Carrillo, 2013). Como refiere Carrillo (2013), el constructo de inteligencia ahora ha sido relegado, debido a la imposibilidad de tomar otros referentes de medida de inteligencia, además del coeficiente intelectual, y por la asequibilidad terapéutica que proporcionan tanto el constructo de la conducta adaptativa como el de la calidad de vida a la hora de realizar un diagnóstico y diseñar una intervención, además de contar con un nuevo clasificador internacional de la discapacidad (OPS-CIF) con una perspectiva ecológica y multidimensional, orientada al desarrollo de intervenciones centrada en las fortalezas (Wehmeyer et al., 2010).

Investigaciones cualitativas (Ali, Strydom, Hassiotis, Williams \& King, 2008) demuestran que al promover los derechos, independencia e inclusión social las personas con discapacidad intelectual, estas siguen experimentando abuso y rechazo y, al ser conscientes del rechazo y de sus consecuencias sociales, intentan ocultar su discapacidad como una forma de evitar la estigmatización. El estigma que las personas con discapacidad intelectual perciben de su entorno se relaciona con mayores niveles de distrés psicológico y con menores niveles de calidad de vida (Ali, King, Strydom \& Hassiotis, 2015).

Algunos autores han llamado la atención sobre el poco interés que las investigaciones sobre salud pública muestran por el estudio de personas con discapacidad intelectual (Brooker et al., 2015). Aunado 
a esto, uno de los grandes obstáculos para el desarrollo de la investigación en el campo de la discapacidad y rehabilitación en el Perú, es la escasez de escalas y/o instrumentos de medición sobre discapacidad intelectual, que reúnan criterios de validez y confiabilidad. Los criterios de evaluación de la discapacidad intelectual no solo consideran la valoración del desarrollo cognitivo, sino también de las habilidades adaptativas (Tenorio, Arroyo, Bunster \& Rosas, 2013). Sin embargo, en la práctica es común observar que se priorice y resalte el valor del coeficiente intelectual (CI) como si los problemas de las personas con discapacidad intelectual fueran solo cognitivos.

Esto conduce a la improvisación e incremento de errores profesionales, que afectan y limitan los procesos de inclusión educativa, social y, por ende, laboral, en desmedro de una evaluación que incluya las habilidades adaptativas para la precisión diagnóstica de la discapacidad intelectual (Tassé, Luckasson \& Schalock, 2016). Esta situación también se ve afectada ante la resistencia en los cambios de paradigmas con respecto a los nuevos enfoques en el abordaje. Si bien la estimación del funcionamiento intelectual es una variable necesaria, no es única, ya que las nuevas perspectivas buscan generar actualmente un diagnóstico que permita definir la intensidad de los apoyos para garantizar la verdadera inclusión social (Tenorio et al., 2013).

La evaluación de la calidad de vida de las personas con discapacidad intelectual está basada en el modelo teórico propuesto por Schalock y Verdugo (Tamarit, 2006), el cual considera ocho dimensiones: bienestar emocional, relaciones interpersonales, desarrollo personal, bienestar material, bienestar físico, autodeterminación, inclusión social y derechos.

Verdugo, Gómez y Arias (2007) realizan un estudio con el objetivo de obtener las propiedades psicométricas de la Escala Integral de Calidad de Vida, dirigida a personas con discapacidad intelectual desde una perspectiva objetiva y subjetiva, siendo la escala objetiva respondida por un observador externo y la escala subjetiva por la misma persona con discapacidad intelectual, en Salamanca y Valladolid. El estudio consistió inicialmente en la construcción de los ítems; para la Escala Objetiva fueron más de 100 ítems, siendo reducida a 38 ítems luego 
de realizar procedimientos básicos para el análisis de los ítems y para la Escala Subjetiva de 300 ítems se redujo a 70. En el estudio piloto, fueron evaluadas 205 personas con discapacidad intelectual, a través de 23 profesionales que trabajaban directamente con personas con discapacidad intelectual, obteniendo un Alpha de Cronbach de .81 y .87, para la Escala Objetiva y Subjetiva respectivamente.Se realizaron, además, procedimientos de validez de constructo y validez de criterio, obteniendo correlaciones significativas.

Verdugo, Gómez, Arias, y Schalock (2009), en una muestra de 861 adultos con discapacidad intelectual, estudian la medida de consistencia interna, con un coeficiente Alfa de Cronbach de .80 para la escala total y valores entre .49 y .74 en las dimensiones. Su evidencia de validez por contenido fue evaluada mediante criterio de jueces expertos, obteniendo coeficientes Krippendorff's Alpha y Kappa adecuados. La evidencia de validez por criterio se obtuvo correlacionando la respuesta mediante una pregunta a los cuidadores sobre la Valoración general de la calidad de vida del evaluado, obteniendo correlaciones positivas, pero de escasa magnitud (entre .20 y .25). En la Escala Objetiva, se realizó un Análisis Factorial reportando un ajuste coherente en los cinco factores.

Gómez et al. (2010) desarrollaron otro estudio sobre la calidad de vida en una muestra de 681 adultos con discapacidad intelectual, procedentes de Argentina, Brasil y Colombia, utilizando la Escala Integral: versión Objetiva y Subjetiva de la Calidad de Vida. En la versión Objetiva, se analizó la consistencia interna por países, obteniendo un coeficiente Alfa de Cronbach de .80 para Argentina, de .66 para Brasil y de .91 en Colombia. Se encontraron diferencias significativas en función de los países, $\chi_{(2)}^{2}=77.82, p<.001$. En la versión subjetiva, el coeficiente Alfa de Cronbach fue de .89, encontrando diferencias significativas en todos los países, $\chi_{(2)}^{2}=9.78, p=.007$, en Argentina el coeficiente fue de .90; en Brasil, de .85; y en Colombia, de .89.

Domínguez y Villegas (2012) en Lima, Perú, realizaron un estudio para evaluar la validez de contenido de la Escala Integral de Calidad de Vida: Versión Objetiva en personas con discapacidad intelectual, por medio de la valoración de 10 jueces expertos. Los resultados, mediante 
el coeficiente $\mathrm{V}$ de Aiken, indicaron un amplio grado de acuerdo entre los jueces con intervalos de confianza superiores a .50, excepto en un ítem referido a la Escala de Autodeterminación que se procedió a eliminar.

Posteriormente, Domínguez (2015), realizó el análisis factorial confirmatorio de la misma versión, en una muestra de 102 personas con discapacidad intelectual con edades comprendidas entre los 18 y 58 años. Se reporta varios procedimientos estadísticos con índices de ajuste y que permiten demostrar que las evidencias de validez por constructo de la escala que no son concluyentes, sugiriendo la necesidad de seguir investigando acerca de las propiedades psicométricas de la presente escala.

Dado el incremento de este tipo de discapacidad en la población peruana, se hace necesario proponer la construcción de escalas de medición y/o adaptación de las mismas, teniendo en cuenta el rigor metodológico de los procedimientos más básicos de validez y confiabilidad, a fin de recomendar éticamente el uso apropiado de las mismas. En esta perspectiva, el presente estudio analiza las propiedades psicométricas de la escala en su versión objetiva, teniendo en cuenta que para adaptar instrumentos de medición en población con discapacidad intelectual es estrictamente necesario iniciar con ajustes de tipo semántico, de acuerdo a la realidad sociocultural de procedencia (Escobar-Pérez y Cuervo-Martínez, 2008).

\section{Método}

Se realizó una investigación de tipo instrumental, referido al desarrollo, adaptación y estudio de las propiedades psicométricas de un test (Ato, López \& Benavente, 2013; Montero \& León, 2007).

\section{Participantes}

El muestreo fue de tipo intencional, se seleccionó a 15 familias con presencia de una persona con discapacidad intelectual, de las cuales 
se escogieron a 3 miembros del contexto familiar, siendo comúnmente la madre la cuidadora principal. Los demás miembros del hogar fueron, en algunos casos, el padre, hermano u otro familiar, todos mayores de edad, obteniendo un total de 45 aplicaciones de la Escala Objetiva. Las edades de los jóvenes oscilaron entre 18 a 33 ańos, de los cuales 9 fueron mujeres y 6 varones.

\section{Instrumentos}

Se utilizó la Escala Integral de la Calidad de Vida de Personas con Discapacidad Intelectual versión Objetiva de Verdugo et al. (2009), con evidencias de validez de contenido, criterio, constructo y confiabilidad por consistencia interna. Para fines del presente estudio, se utilizó únicamente la Escala Objetiva, que recoge aspectos observables de la calidad de vida a través de la valoración de una persona externa (cuidador) que lo conozca bien. Cuenta con 23 ítems y un formato de respuesta de tipo dicotómico, teniendo como dimensiones: autodeterminación, bienestar emocional y físico, bienestar material, inclusión social y bienestar laboral.

\section{Procedimiento}

Para la recolección de los datos se contactó con 15 familias procedentes del Cercado de Lima, explicando el objetivo del estudio y solicitando el Consentimiento Informado de cada una de ellas para que sea cumplimentada por cada miembro del hogar.

Los datos de la evaluación se estructuraron en una hoja de cálculo en Microsoft Excel 2010. La evidencia de validez por contenido fue efectuada mediante 10 jueces expertos, quienes cumplían los criterios básicos de selección como formación académica, experiencia en la temática y reconocimiento de la comunidad en la misma. Se realizó el análisis en un programa en lenguaje Visual Basic para el cálculo del coeficiente V de Aiken y sus intervalos de confianza (Merino \& Livia, 2009), lo cual mide 
mide el grado en que los ítems reflejen el contenido de las áreas implicadas en el constructo en una proporción adecuada (Escurra, 1989), en términos de claridad, coherencia, relevancia del ítem y suficiencia de la dimensión, con respecto a un dominio de contenido. El acuerdo entre jueces se obtuvo por medio de juicios graduados a diferencia de juicios dicotómicos (Escobar-Pérez \& Cuervo-Martínez, 2008).

Para el análisis de confiabilidad inter-observador por el coeficiente Alfa de Krippendroff (Hayes \& Krippendorff, 2007), que cuantifica el grado de acuerdo entre 3 observadores para cada ítem, se utilizó el programa estadístico R versión 3.2.0 (2015), mediante los paquetes base e "irr".

Las modificaciones de los ítems se hicieron sobre la base de las opiniones de los jueces expertos y los resultados del V de Aiken y el Alfa de Krippendorff, siguiendo los lineamientos de adaptación semántica propuestas en las directrices para la traducción y adaptación de los tests (Muñiz, Elosua \& Hambleton, 2013) teniendo en cuenta, además, los siguientes parámetros:

- Si el valor de V de Aiken es mayor de .80 y Alfa de Krippendorff es mayor de .70 en todos los indicadores se mantendría el ítem.

- Si el valor de V de Aiken es menor de .80 y el Alfa de Krippendorff es menor de .40 en todos los criterios, se descartaba el ítem.

- Si el valor de V de Aiken es menor de .80 en uno o dos de los criterios y mayor de .40 en Alfa de Krippendorff, se hacían las correcciones y ajustes al ítem.

\section{Resultados}

En la Tabla 1 se presentan los ítems que fueron modificados de acuerdo a las observaciones de los jueces expertos, al coeficiente $\mathrm{V}$ de Aiken y a los resultados del análisis de confiabilidad inter-observadores Alfa de Krippendorff realizado ítem por ítem. 
Validez de contenido y confiabilidad inter-observadores de la Escala Integral... / Boluarte y Tamari

\section{Tabla 1}

Análisis de validez de contenido y confiabilidad inter-observadores en la dimensión Autodeterminación

\begin{tabular}{|c|c|c|c|c|c|}
\hline & Categoría & $\begin{array}{l}\text { V de } \\
\text { Aiken }\end{array}$ & IC 95\% & $\begin{array}{l}\text { Alfa de } \\
\text { Krippen- } \\
\text { dorff }\end{array}$ & Nuevos ítems \\
\hline \multirow{3}{*}{$\begin{array}{l}\text { 1. Manifiesta inte- } \\
\text { reses y objetivos } \\
\text { propios y hace } \\
\text { planes para alcan- } \\
\text { zarlos. }\end{array}$} & Claridad & .83 & {$[.64-.93]$} & \multirow[b]{3}{*}{.62} & \multirow{3}{*}{$\begin{array}{l}\text { Se desglosa en } 2 \text { ítems: } \\
\text { 1.Expresa sus metas, } \\
\text { objetivos e intereses } \\
\text { propios } \\
\text { 2.Organiza y programa } \\
\text { sus actividades }\end{array}$} \\
\hline & Coherencia & .96 & {$[.83-.99]$} & & \\
\hline & Relevancia & 1 & {$[.89-1]$} & & \\
\hline \multirow{3}{*}{$\begin{array}{l}\text { 2. Se mueve de } \\
\text { forma independiente } \\
\text { por su comunidad }\end{array}$} & Claridad & .83 & {$[.64-.93]$} & \multirow{3}{*}{.55} & \multirow{3}{*}{$\begin{array}{l}\text { Se moviliza de forma } \\
\text { independiente por su } \\
\text { barrio }\end{array}$} \\
\hline & Coherencia & 1 & {$[.89-1]$} & & \\
\hline & Relevancia & 1 & {$[.89-1]$} & & \\
\hline \multirow{3}{*}{$\begin{array}{l}\text { 5. Si alguien le } \\
\text { robara sabría cómo } \\
\text { denunciarlo a la } \\
\text { policía }\end{array}$} & Claridad & .76 & {$[.59-.88]$} & \multirow{3}{*}{.08} & \multirow{3}{*}{ Eliminado } \\
\hline & Coherencia & .76 & {$[.59-.88]$} & & \\
\hline & Relevancia & .057 & {$[.39-.73]$} & & \\
\hline \multirow{3}{*}{$\begin{array}{l}\text { 6. Le dejan votar } \\
\text { cuando hay } \\
\text { elecciones }\end{array}$} & Claridad & .76 & {$[.59-.88]$} & \multirow{3}{*}{.61} & \multirow{3}{*}{$\begin{array}{l}\text { Vota cuando hay } \\
\text { elecciones }\end{array}$} \\
\hline & Coherencia & .96 & {$[.83-.99]$} & & \\
\hline & Relevancia & .96 & {$[.83-.99]$} & & \\
\hline \multirow{3}{*}{$\begin{array}{l}\text { 7. Participa en } \\
\text { asociaciones de } \\
\text { su comunidad: de } \\
\text { vecinos, del barrio, } \\
\text { parroquiales, etc. } \\
\text { y en actividades } \\
\text { de ocio como: } \\
\text { conciertos, espec- } \\
\text { táculos, cines, } \\
\text { cafeterías, parques, } \\
\text { etc. }\end{array}$} & Claridad & .73 & {$[.56-.86]$} & & \multirow{3}{*}{$\begin{array}{l}\text { Pasa a Dimensión } \\
\text { Inclusión Social: } \\
\text { 1. Participa en grupos } \\
\text { de su comunidad: de } \\
\text { vecinos, del barrio, } \\
\text { parroquiales. } \\
\text { 2. Participa en } \\
\text { actividades culturales/ } \\
\text { recreativas: concierto, } \\
\text { espectáculos, cines, } \\
\text { cafeterías, parques, etc. }\end{array}$} \\
\hline & Coherencia & .90 & {$[.74-.96]$} & .45 & \\
\hline & Relevancia & .90 & {$[.74-.96]$} & & \\
\hline \multirow{5}{*}{$\begin{array}{l}\text { 8. Mantiene su } \\
\text { habitación ordenada } \\
\text { y limpia }\end{array}$} & Claridad & .86 & {$[.70-.95]$} & \multirow{3}{*}{.42} & \multirow{3}{*}{$\begin{array}{l}\text { Ordena su habitación a } \\
\text { su gusto }\end{array}$} \\
\hline & Coherencia & .93 & {$[.79-.98]$} & & \\
\hline & Relevancia & .96 & {$[.83-.99]$} & & \\
\hline & Suficiencia & .80 & {$[.63-.90]$} & & \\
\hline & & & & & .37 \\
\hline
\end{tabular}

Jueces $=10$ 
En la dimensión Autodeterminación, el ítem $1(\alpha=.61)$ tiene dos proposiciones, por lo que se decide desglosar en 2 ítems describiendo del siguiente modo: "Expresa sus metas, objetivos e intereses propios" y "Organiza y programa sus actividades"; el ítem 5 es eliminado por obtener una baja confiabilidad inter-observadores $(\alpha=.07)$; el ítem 7 se desglosa en dos, observando baja confiabilidad $(\alpha=.45)$ y se incluyen en la Dimensión Inclusión Social, mientras que los ítems 2 , 6 y 8 se realizan precisiones semánticas.

\section{Tabla 2}

Análisis de validez de contenido y confiabilidad inter-observadores en la dimensión Inclusión Social

\begin{tabular}{|c|c|c|c|c|c|}
\hline & Categoría & $\begin{array}{l}\text { V de } \\
\text { Aiken }\end{array}$ & IC $95 \%$ & $\begin{array}{c}\text { Alfa de } \\
\text { Krippen- } \\
\text { dorff }\end{array}$ & Nuevos ítems \\
\hline \multirow{3}{*}{$\begin{array}{l}\text { 9. Tiene amigos } \\
\text { con los que realiza } \\
\text { actividades de su } \\
\text { agrado }\end{array}$} & Claridad & .9 & {$[.74-.96]$} & \multirow{3}{*}{.73} & \multirow{3}{*}{$\begin{array}{l}\text { Realiza actividades de } \\
\text { agrado con sus amigos }\end{array}$} \\
\hline & Coherencia & 1 & {$[.89-1]$} & & \\
\hline & Relevancia & 1 & {$[.89-1]$} & & \\
\hline \multirow{3}{*}{$\begin{array}{l}\text { 10. Es tratado por } \\
\text { los demás como } \\
\text { cualquier otra } \\
\text { persona }\end{array}$} & Claridad & .83 & {$[.64-.93]$} & & \multirow{3}{*}{$\begin{array}{l}\text { Se desglosa en } 2 \text { ítems: } \\
\text { 1. Las personas en casa } \\
\text { lo tratan igual que los } \\
\text { demás. } \\
\text { 2. Las personas del } \\
\text { barrio y/o comunidad } \\
\text { lo tratan igual que los } \\
\text { demás. }\end{array}$} \\
\hline & Coherencia & .93 & {$[.79-.98]$} & .24 & \\
\hline & Relevancia & .93 & {$[.79-.98]$} & & \\
\hline \multirow{5}{*}{$\begin{array}{l}\text { 11. Los demás } \\
\text { respetan su intimidad } \\
\text { (privacidad) }\end{array}$} & Claridad & .90 & {$[.74-.96]$} & \multirow{3}{*}{.26} & \multirow{3}{*}{$\begin{array}{l}\text { Pasa a Dimensión } \\
\text { Autodeterminación: } \\
\text { Otras personas deciden } \\
\text { sobre su vida personal. }\end{array}$} \\
\hline & Coherencia & .93 & {$[.79-.98]$} & & \\
\hline & Relevancia & .83 & {$[.64-.93]$} & & \\
\hline & Suficiencia & .60 & {$[.42-.75]$} & & \\
\hline & & & & & .29 \\
\hline
\end{tabular}

Jueces $=10$

En la dimensión Inclusión Social, el ítem 10 presenta bajo coeficiente Alfa de Krippendorff ( $\alpha=.24)$ desglosándolo en dos ítems expresado como: "Las personas en casa lo tratan igual que los demás" y "Las personas del barrio y/o comunidad lo tratan igual que los demás". 
Validez de contenido y confiabilidad inter-observadores de la Escala Integral... / Boluarte y Tamari

El ítem 11 se agrega a la dimensión Autodeterminación, expresado del siguiente modo: "Otras personas deciden sobre su vida personal".

\section{Tabla 3}

Análisis de validez de contenido y confiabilidad inter-observadores en la dimensión Bienestar Laboral

\begin{tabular}{|c|c|c|c|c|c|}
\hline & Categoría & $\begin{array}{l}\mathrm{V} \text { de } \\
\text { Aiken }\end{array}$ & IC 95\% & $\begin{array}{l}\text { Alfa de } \\
\text { Krippen- } \\
\text { dorff } \\
\end{array}$ & Nuevos ítems \\
\hline \multirow{3}{*}{$\begin{array}{l}\text { 12. Realiza su } \\
\text { trabajo o actividades } \\
\text { de forma responsable } \\
\text { y competente }\end{array}$} & Claridad & .87 & {$[.70-.95]$} & & \multirow{3}{*}{$\begin{array}{l}\text { Se desglosa en } 2 \text { ítems: } \\
\text { 1. En el Taller/ } \\
\text { Trabajo cumple con las } \\
\text { indicaciones dadas } \\
\text { 2. Sus labores en el } \\
\text { taller/trabajo las realiza } \\
\text { a tiempo }\end{array}$} \\
\hline & Coherencia & .73 & {$[.56-.86]$} & & \\
\hline & Relevancia & .77 & {$[.59-.88]$} & .16 & \\
\hline \multirow{3}{*}{$\begin{array}{l}\text { 13. Mantiene una } \\
\text { buena relación con } \\
\text { sus compańeros }\end{array}$} & Claridad & .90 & {$[.74-.96]$} & \multirow{3}{*}{.35} & \multirow{3}{*}{$\begin{array}{l}\text { Sus compañeros lo } \\
\text { aceptan con agrado. }\end{array}$} \\
\hline & Coherencia & .73 & {$[.56-.86]$} & & \\
\hline & Relevancia & .83 & {$[.64-.93]$} & & \\
\hline \multirow{3}{*}{$\begin{array}{l}\text { 14. Está } \\
\text { compenetrado con } \\
\text { sus compańeros de } \\
\text { trabajo o del taller }\end{array}$} & Claridad & .73 & {$[.56-.86]$} & \multirow{3}{*}{.35} & $\begin{array}{l}\text { Se elimina el ítem por } \\
\text { considerarlo similar al } \\
\text { anterior, se adicionan }\end{array}$ \\
\hline & Coherencia & .37 & {$[.22-.54]$} & & $\begin{array}{l}2 \text { ítems: } \\
\text { 1. En el Taller/Trabajo } \\
\text { realiza actividades } \\
\text { remuneradas }\end{array}$ \\
\hline & Relevancia & .63 & {$[.45-.78]$} & & $\begin{array}{l}\text { 2. En el Taller/Trabajo } \\
\text { recibe pago justo de } \\
\text { acuerdo a ley }\end{array}$ \\
\hline
\end{tabular}

Suficiencia $\quad .50 \quad[.33-.69]$

.29

Jueces $=10$

En la dimensión Bienestar Laboral, el ítem 12 presenta bajo nivel de coherencia y relevancia $(V=.73, .77)$ y un Alfa de Krippendorff muy bajo $(\alpha=.16)$ por lo que se decide desglosar en dos ítems: "En el Taller/ Trabajo cumple con las indicaciones dadas" y "Sus labores en el Taller/ Trabajo las realiza a tiempo". Se realiza un cambio semántico al ítem 13; el ítem 14 es eliminado y se adicionan dos ítems: "En el Taller/Trabajo realiza actividades remuneradas" y "En el Taller/Trabajo recibe pago justo de acuerdo a Ley". 


\section{Tabla 4}

Análisis de validez de contenido y confiabilidad inter-observadores en la dimensión Bienestar Material

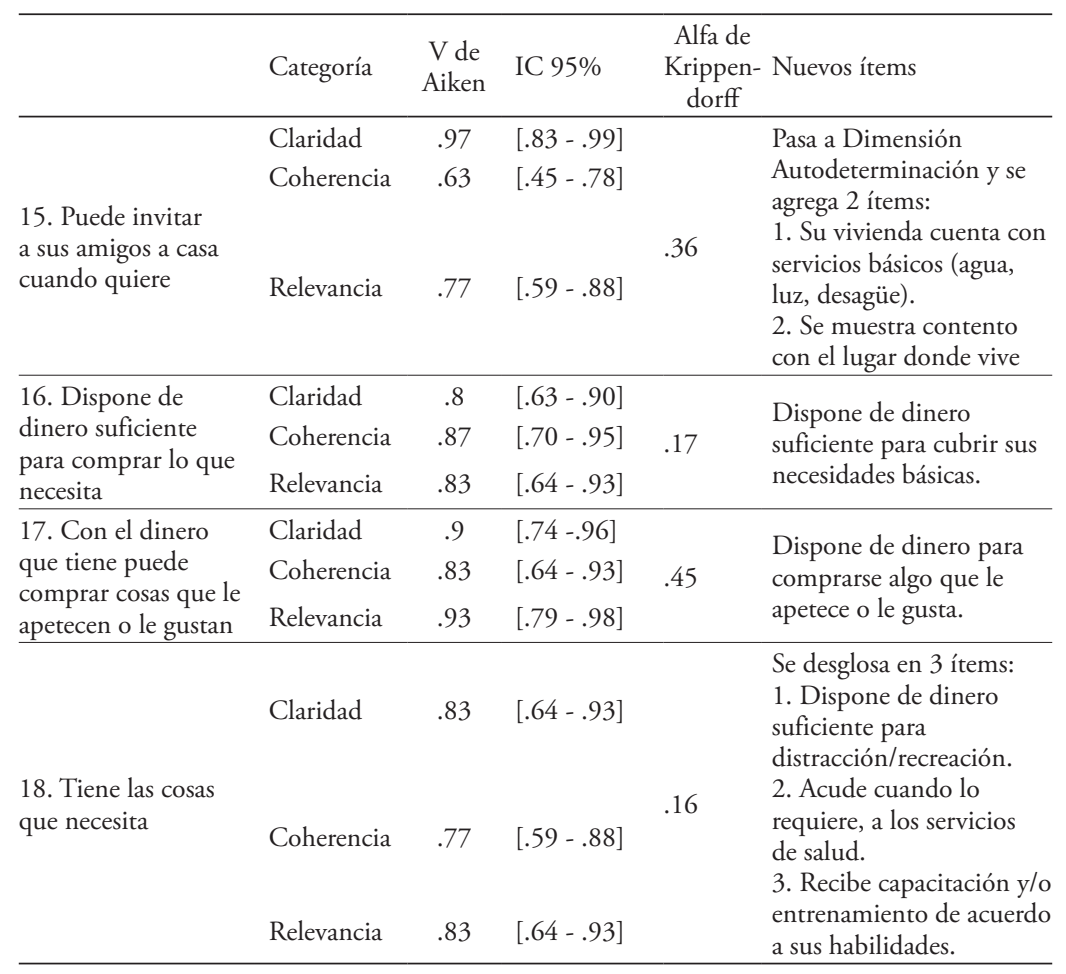

Suficiencia $\quad .70 \quad[.52-.83]$

Jueces $=10$

En la dimensión Bienestar Material, el ítem 15 se incluye en la dimensión Autodeterminación, agregando dos ítems relacionados: "Su vivienda cuenta con servicios básico (agua, luz, desagüe)" y "Se muestra contento con el lugar donde vive". En el ítem 16, se realiza precisión semántica por presentar bajo coeficiente Alfa de Krippendorff $(\alpha=.17)$; el ítem 17 presenta un bajo nivel de confiabilidad $(\alpha=.45)$, 
Validez de contenido y confiabilidad inter-observadores de la Escala Integral... / Boluarte y Tamari

por lo que se realizaron precisiones semánticas. Se elimina el ítem 18 debido a su coeficiente Alfa de Krippendorff muy bajo $(\alpha=.16)$, agregándose tres ítems: "Dispone de dinero suficiente para distracción/ recreación", "Acude cuando o requiere, a los servicios de salud", "Recibe capacitación y/o entrenamiento de acuerdo a sus habilidades".

\section{Tabla 5}

Análisis de validez de contenido y confiabilidad inter-observadores de las dimensiones Bienestar Emocional y Bienestar Físico

\begin{tabular}{|c|c|c|c|c|c|}
\hline & Categoría & $\begin{array}{l}\mathrm{V} \text { de } \\
\text { Aiken }\end{array}$ & IC $95 \%$ & $\begin{array}{l}\text { Alfa de } \\
\text { Krippen- } \\
\text { dorff }\end{array}$ & Nuevos ítems \\
\hline \multirow{3}{*}{$\begin{array}{l}\text { 19. Se muestra } \\
\text { satisfecho consigo } \\
\text { mismo }\end{array}$} & Claridad & .90 & {$[.74-.96]$} & \multirow{3}{*}{.44} & Bienestar Emocional: \\
\hline & Coherencia & .93 & {$[.79-.98]$} & & Se acepta mostrándose contento \\
\hline & Relevancia & 1 & {$[.89-1]$} & & con su propia persona. \\
\hline \multirow{3}{*}{$\begin{array}{l}\text { 20. Se muestra } \\
\text { satisfecho con su } \\
\text { vida }\end{array}$} & Claridad & .83 & {$[.64-.93]$} & \multirow{3}{*}{.39} & Bienestar Emocional: \\
\hline & Coherencia & .83 & {$[.64-.93]$} & & Se muestra contento con su \\
\hline & Relevancia & .90 & {$[.74-.96]$} & & vida. \\
\hline \multirow{3}{*}{$\begin{array}{l}\text { 21. Habitualmente } \\
\text { está alegre y de } \\
\text { buen humor }\end{array}$} & Claridad & .90 & {$[.74-.96]$} & \multirow{3}{*}{.52} & Bienestar Emocional: \\
\hline & Coherencia & .87 & {$[.70-.95]$} & & Se agrega un ítem: \\
\hline & Relevancia & .90 & {$[.74-.96]$} & & $\begin{array}{l}\text { Muestra motivación y entusias- } \\
\text { mo para realizar actividades. }\end{array}$ \\
\hline \multirow{3}{*}{$\begin{array}{l}\text { 22. Su estado } \\
\text { general de salud } \\
\text { es bueno }\end{array}$} & Claridad & .97 & {$[.83-.99]$} & \multirow{3}{*}{.16} & \multirow{3}{*}{ Bienestar Físico } \\
\hline & Coherencia & .97 & {$[.83-.99]$} & & \\
\hline & Relevancia & .93 & {$[.79-.98]$} & & \\
\hline \multirow{7}{*}{$\begin{array}{l}\text { 23. Su estado de } \\
\text { salud le permite } \\
\text { llevar una actividad } \\
\text { normal }\end{array}$} & Claridad & .83 & {$[.64-.93]$} & \multirow{5}{*}{.33} & Bienestar Físico: \\
\hline & Coherencia & 1 & {$[.89-1]$} & & Se desglosa en 2 ítems: \\
\hline & & & & & $\begin{array}{l}\text { 1. Su estado de salud le permite } \\
\text { realizar sus actividades de casa }\end{array}$ \\
\hline & Relevancia & .97 & {$[.83-.99]$} & & $\begin{array}{l}\text { 2. Su estado de salud le permi- } \\
\text { te realizar actividades físicas o } \\
\text { deporte }\end{array}$ \\
\hline & & & & & $\begin{array}{l}\text { Se agrega un ítem: Recibe una } \\
\text { nutrición balanceada }\end{array}$ \\
\hline & Suficiencia & 1 & [. $89-1]$ & \multicolumn{2}{|c|}{$\begin{array}{l}\text { Se separa en } 2 \text { dimensiones: Bienestar } \\
\text { Físico y Bienestar Emocional. }\end{array}$} \\
\hline & & & & & .30 \\
\hline
\end{tabular}


De acuerdo a los resultados encontrados en la validez de contenido y la confiabilidad inter-observadores, la última dimensión Bienestar Emocional y Físico se separa en 2 dimensiones. El ítem 19, correspondiente a la Dimensión Bienestar Emocional, es redactado del siguiente modo: "se acepta mostrándose contento con su propia persona", el ítem 20 se describe como: "se muestra contento con su vida", debido a que su nivel de confiabilidad es bajo $(\alpha=.39)$. Se agrega el siguiente ítem:" Muestra motivación y entusiasmo para realizar actividades". El ítem 23 presenta un Alfa de Krippendorff bajo $(\alpha=.33)$, por lo que se decide desglosar en dos ítems: "Su estado de salud le permite realizar sus actividades de casa"," Su estado de salud le permite realizar actividades físicas o deporte "y se agrega un ítem: "Recibe una nutrición balanceada".

Finalmente se reporta la confiabilidad Alfa de Krippendorff de la escala total indicando un coeficiente de .58, por debajo del nivel esperado (Alonso, Volkens \& Gómez, 2012).

\section{Discusión}

Considerando la validez y confiabilidad como requisitos de todo instrumento de medición, que van desde niveles básicos hasta niveles avanzados con procesos metodológicos rigurosos se considera pertinente la propuesta de una escala breve que pueda reflejar con objetividad y precisión todos los indicadores más relevantes relacionados a la percepción de la calidad de vida de la persona con discapacidad intelectual. Este es el caso de la escala utilizada en el presente estudio, cuya validación original se realizó en España (Verdugo et al., 2007), se encontró como antecedentes en nuestro medio los estudios psicométricos de Domínguez (2012, 2015). Se requiere continuar con estudios cuya revisión y análisis exhaustivo nos permita recomendar su uso en la población peruana, empezando desde procedimientos de validez aparente y de contenido de la mano con procedimientos de confiabilidad, debido a que ambos demostrarán la calidad que posee el instrumento (Urrutia, Barrios, Gutiérrez \& Mayorga, 2014), de manera 
que se garantice su correspondencia con la realidad sociocultural, las diferencias psicológicas de la población diana y su aplicabilidad práctica (Muñiz et al., 2013).

En el proceso de construcción y/o adaptación de un instrumento de medición, la validez de contenido representa el primer nivel de validez que da cuenta de la correspondencia entre la teoría y la característica del fenómeno observado (Urrutia et al., 2014). En tal sentido, los resultados de la presente investigación ponen en evidencia la necesidad de incrementar estudios que partan desde la construcción de escalas de medición hasta la adaptación de las mismas en caso de escalas validadas en otras realidades.

Los resultados indican la necesidad de realizar ajustes desde el punto de vista semántico, lingüístico y cultural de la Escala integral de Calidad de Vida: Versión Objetiva, observando un acuerdo substancial sobre la baja validez de contenido de los ítems en términos de claridad, coherencia, relevancia y suficiencia, y un bajo nivel de confiabilidad inter-observadores, proponiendo una escala breve y adaptada a las características socioculturales, psicológicas y lingüísticas de personas con discapacidad intelectual procedentes de centros de habilitación de Lima.

De acuerdo a las sugerencias de los Jueces Expertos, fue necesario realizar ajustes a los indicadores a fin de que representen a la dimensión, teniendo especial cuidado en la definición conceptual de cada una, derivando en una propuesta basada en la utilidad de los datos obtenidos, no solo de la validez de contenido con el V de Aiken y sus intervalos de confianza, sino también con los datos del coeficiente Alfa de Krippendorff.

Teniendo en cuenta que el Alfa de Krippendorff es una medida estándar de fiabilidad que se utiliza para cuantificar el grado de acuerdo entre observadores, independientemente del número de observadores, niveles de medición, tamaño de las muestras y la presencia o ausencia de los datos que faltan (Hayes \& Krippendorff, 2007; Gwet, 2011), otros estudios demuestran el uso de Alfa de Krippendorff para verificar índice de acuerdo entre jueces, con el fin de valorar validez de contenido 
(Verdugo et al., 2007), cuyo resultado resulta ser confuso ya que no se puede saber si un acuerdo optimo estaría reflejando un adecuada o baja validez de contenido del ítem.

Se considera que el coeficiente $\mathrm{V}$ de Aiken es el estadístico más pertinente para valorar la validez de contenido (Escurra, 1989), cuyos valores se esperan que sean superiores a .70 para que el ítem sea considerado valido (Merino \& Livia, 2009), mientras que el Alfa de Kriperdorf se utiliza como índice de acuerdo para confiabilidad interobservadores, es decir, para verificar la estabilidad de los datos (Hayes \& Krippendorff, 2007; Gwet, 2011; Krippendorff, 2011).

Los resultados encontrados contradicen lo reportado por Domínguez (2012), quien afirma adecuados niveles en el Coeficiente V de Aiken en la totalidad de los ítems, a excepción de los ítems 3, 4 y 5, coincidiendo con el ítem 5 con un bajo coeficiente $\mathrm{V}$ de Aiken, ( $\mathrm{V}=.59)$ Domínguez (2012), obteniendo también en el presente estudio un coeficiente de Aiken de .57 , en cuanto a la relevancia del ítem y un Alfa de Krippendorf de .08 que indica nula confiabilidad, siendo el ítem eliminado, en ambos casos.

La confiabilidad inter-observadores es el grado de concordancia entre dos o más personas, es decir, hasta qué punto ambos coinciden en su medición. Para determinar el grado de concordancia interobservador, se dispone de una herramienta estadística frecuentemente utilizada, que es el coeficiente Kappa ( $\kappa$ ) cuando estos son de tipo categórico (Cerda \& Villarroel, 2008); sin embargo, el Alfa de Krippendorff es el más versátil y recomendado últimamente, el mismo que puede utilizarse para cualquier tipo de escala, con distribución normal o no y con dos o más observadores (Krippendorff, 2011), reflejando la fuerza de la concordancia entre dos o más observadores.

En el análisis dimensional, la Autodeterminación se entiende como una característica de personalidad que permite asumir un rol activo en la toma de decisiones sobre su vida personal, libre de influencias externas (Pascual-García, Garrido-Fernández \& Antequera-Jurado, 2014). En el caso de personas con discapacidad intelectual, se puede observar que son los cuidadores o profesionales quienes deciden sobre 
su vida, su futuro, asumiendo una actitud sobreprotectora que limita oportunidades de desarrollo personal; esta situación predispone al joven a desarrollar un sentimiento de minusvalía, desconfianza e inseguridad en sí mismo, lo que se demuestra con solo un $12.6 \%$ de inserción laboral en esta muestra.

Las personas con discapacidad intelectual parecen ocupar un segundo plano en la toma de decisiones en lo que afecta su propia calidad de vida. (Pascual-García et al., 2014). En nuestro medio, es común observar violencia física y psicológica, aunque no exista en nuestro medio algún estudio que lo demuestre, suelen ser los cuidadores quienes vulneran sus derechos. Es así que se considera de suma importancia continuar estudios con la Versión Subjetiva, ya que, habiendo sido aplicada conjuntamente con la Escala Objetiva, ya se reportaron indicadores de violencia expresados por los mismos jóvenes, considerando pertinente valorar sus opiniones a pesar del bajo nivel cognitivo.

En la versión de la Escala Objetiva, Domínguez (2014) sugiere cambios semánticos en 12 ítems, siendo uno de ellos eliminado (ítem 5), sin embargo, en el presente estudio, se adicionan ítems en la dimensión inclusión social, bienestar laboral, bienestar material, bienestar emocional y físico, como se muestra en la Tabla 1, además de la rotación de ítems a otras dimensiones.

En la dimensión Inclusión social, entendida como el nivel de participación social en igualdad de oportunidades (Gálvez, 2009), se realizan ajustes y cambios a los ítems, siendo los siguientes: el ítem 9 se realiza cambios semánticos, lo cual coincide con lo reportado por Domínguez (2012), por presentar bajo nivel de claridad (V = .74); el ítem 10, con el enunciado "es tratado por los demás como cualquier otro", se desglosa en 2 ítems, para mayor precisión, como se aprecia en la Tabla 1; mientras que el ítem 11 pasa a la dimensión de autodeterminación, por considerarse el respeto a su privacidad personal un tema de autoafirmación.

Con respecto a la dimensión Bienestar Laboral los resultados obtenidos ameritan el desglose del ítem 12 en dos ítems, debido al bajo nivel de confiabilidad. El ítem 13 es modificado desde el punto de vista 
semántico acorde al léxico comúnmente empleado en nuestro medio. Se elimina el ítem 14 por considerarse similar al anterior y se adicionan dos ítems relacionados a las condiciones laborales.

La dimensión Bienestar Material, relacionada a la satisfacción de sus necesidades básicas, considerando, no solo la capacidad adquisitiva sino también la accesibilidad a los servicios de salud y de capacitación, de manera que se adicionan ítems relacionado a esos indicadores.

En la dimensión Bienestar emocional y físico, se considera necesario dividirla en 2 dimensiones para garantizar su comprensión y amplitud de información, tanto de su estado de salud física como de su estado emocional.

Uno de los aspectos importantes con respecto a la calidad de los datos, es la confiabilidad, de manera que pueda reflejar la idiosincrasia de la unidad de análisis, es decir, reflejar con exactitud las propiedades de los fenómenos estudiados (Hayes \& Krippendorff, 2007). Observamos que, en algunos estudios psicométricos, se fuerzan los análisis estadísticos con el fin de obtener coeficientes óptimos, desvirtuando la esencia teórica y evidencia empírica de la variable de interés. Siendo la versión original de tipo dicotómica, se recomienda, como parte de los cambios sugeridos una escala de tipo ordinal con valores que van desde Siempre, Casi siempre, A veces y Nunca.

La literatura relacionada a la confiabilidad y validez de una escala de medición, presenta múltiples propuestas, entre ellos los llamados "coeficientes de fiabilidad", dejando a los investigadores confundidos y en la disyuntiva de no saber qué elegir (Hayes \& Krippendorff, 2007). Es recomendable evaluar con cuidado los criterios técnicos y metodológicos de cada técnica, de manera que puedan ayudar a tomar decisiones acertadas.

Existe la necesidad de continuar con estudios psicométricos para la adaptación de instrumentos de valoración de la calidad de vida con la finalidad de medir, según criterios científicos y de calidad, la labor que se desarrolla tanto a nivel asistencial como con fines de investigación en este grupo poblacional. 
La carencia de instrumentos apropiados para valorar la discapacidad no permite un diagnóstico preciso de las limitaciones funcionales, generando tratamientos imprecisos, genéricos y difusos, prolongando así el tiempo del proceso de rehabilitación, encareciendo los costos, lo que repercute a nivel económico, social y afectivo de cada familia, incrementando la presencia de trastornos asociados y limitando la prevención secundaria de discapacidades asociadas.

Los resultados indican la necesidad de continuar estudios psicométricos en este grupo poblacional, según las características de la realidad social y cultural de personas con discapacidad intelectual procedentes de Centros de Rehabilitación y Entrenamiento a nivel de Lima Metropolitana, impulsando, de esta manera, el desarrollo de instrumentos de medición para la investigación que sean válidos y confiables y permitan no solo precisar enfoques diagnósticos, sino también puedan ser utilizados para la medición de la eficacia de las intervenciones en el campo de la discapacidad y rehabilitación.

Entre las limitaciones encontradas para el desarrollo de la presente investigación, se enmarcaron como parte del procedimiento de confiabilidad inter-observador dificultades de accesibilidad a la muestra y, en algunos casos, la escasa disposición de los familiares a colaborar con la aplicación de las escalas; asimismo, está el hecho de ser una muestra perteneciente solo a instituciones públicas, donde el nivel cultural de los familiares podría interferir con el entendimiento de los ítems. Sin embargo, desde la perspectiva sistémica estas diferencias serían válidas, ya que las interacciones diádicas y tríadicas entre los miembros del contexto familiar son interdependientes (Fairlie \& Frisancho, 1998) y sus percepciones varían a partir de las propias vivencias del sujeto (Vargas, 1994). Además, como el instrumento de medición se basó en aspectos observables de la calidad de vida y en formato dicotómico, y no en un cuestionario, se esperaba que a través de la fiabilidad inter-observador se obtuvieran puntajes relativamente estables, independientemente de quién sea el observador (Rodríguez \& Heredia, 2013). 


\section{Referencias}

Ali, A, Strydom, A., Hassiotis, A., Williams, R. y King, M. (2008). A measure of perceived stigma in people with intellectual disability. The British Journal of Psychiatry, 193(5), 410-415. https://doi. org/10.1192/bjp.bp.107.045823

Ali, A., King, M., Strydom, A. y Hassiotis, A. (2015). Self-reported stigma and symptoms of anxiety and depression in people with intellectual disabilities: Findings from a cross sectional study in England. Journal of Affective Disorders, 187, 224-231. https:// doi.org/10.1016/j.jad.2015.07.046

Alonso, S., Volkens, A. y Gómez, B. (2012). Validez y Fiabilidad. En S. L. Caslon (Ed), Análisis de contenido de textos políticos. Un enfoque cuantitativo (pp. 36-37). Madrid, España: Centro de Investigaciones Sociológicas.

Asociación Americana de Psiquiatría (2014). Manual diagnóstico y estadístico de los trastornos mentales, Quinta edición. Madrid: Panamericana.

Ato, M., López, J. y Benavente, A. (2013). Un sistema de clasificación de los diseños de investigación en psicología. Anales de Psicología, 29(3), 1038-1059. https://doi.org/10.6018/analesps. 29.3.178511

Barlow, D. H. y Durand, V. M. (2015). Abnormal psychology: An integrated approach. Stamford: Cengage Learning.

Boluarte, A., Méndez, J. y Martell, R. (2006). Programa de entrenamiento en habilidades sociales para jóvenes con retraso mental leve y moderado. Mosaico Cient, 3(1), 34-42.

Brooker, K., van Dooren, K., Tseng, C. H., McPherson, L., Lennox, N., Ware, R. (2015). Out of sight, out of mind? The inclusion and identification of people with intellectual disability in public health research. Perspectives in Public Health, 135(4), 204-211. https://doi.org/10.1177/1757913914552583

Carrillo M. (2013). Inteligencia, conducta adaptativa y calidad de vida: interacciones explicativas de la discapacidad intelectual 
y la intervención optimizadora (Tesis Doctoral). Universidad Autónoma de Barcelona. Recuperado de http://ddd.uab.cat/ record/114010

Córdoba, L., Mora, A., Bedoya, A. M. y Verdugo, M. A. (2007). Familias de adultos con discapacidad intelectual en Cali, Colombia, desde el modelo de calidad de vida. Psykhe, 16, 29-42. https:// doi.org/10.4067/S0718-22282007000200003

Curryer, B., Stancliffe, R. J. y Dew, A. (2015). Self-determination: Adults with intellectual disability and their family. Journal of Intellectual and Developmental Disability, 40(4), 394-399. https://doi.org/10.3109/13668250.2015.1029883

Domínguez, S. (2015). Propiedades psicométricas de una escala de calidad de vida para personas adultas con discapacidad intelectual. Psicología. Avances de la Disciplina, 9(1), 29-43. https://doi. org/10.21500/19002386.991

Domínguez, S. y Villegas, G. (2012). Estimación de la validez de contenido de una escala de calidad de vida para personas adultos con discapacidad intelectual. Revista de Psicología de Arequipa, 2(2), 207-219.

Escobar-Pérez, J., Cuervo-Martínez, A. (2008). Validez de contenido y juicio de expertos. Avances en Medición, 6, 27-36

Escurra, M. (1989). Cuantificación de la validez de contenido por criterio de jueces. Revista de Psicología, 6(1-2), 103-111.

Fairlie, A. \& Frisancho, D. (1998). Teoría de las interacciones familiares. Revista de Investigación en Psicología, 1(2), 41-74.

Gálvez, E. (2009). La inclusión social y laboral de las personas con discapacidad intelectual mediante los programas de empleo con apoyo. REOP, 20(2), 135-146.

Giné, C. (2004). Servicios y calidad de vida para las personas con discapacidad intelectual. Revista Española sobre Discapacidad Intelectual, 35(2), 18-28.

Gómez, L., Verdugo, M. y Arias, B. (2010, mayo). Calidad de vida en personas con discapacidad en Argentina, Colombia y Brasil: resultados de la Escala Integral. Ponencia presentada en el II Congreso Iberoamericano sobre Síndrome de Down, Granada, España. 
Gómez, L., Verdugo, M. y Arias, B. (2010). Calidad de vida individual: Avances en su conceptualización y retos emergentes en el ámbito de la discapacidad. Psicología Conductual, 18(3), 453-472.

Gwet, K. K. (2011). On the Krippendorff's alpha coefficient. Recuperado de http://www.agreestat.com/research_papers/onkrippendorff alpha.pdf

Hayes, A. y Krippendorff, K. (2007). Answering the call for a standard reliability measure for coding data. Communication Methods and Measures, 1, 77-89. https://doi.org/10.1080/1931245070 9336664

Krippendorff, K. (2011). Computing Krippendorff's alpha-reliability. Recuperado de http://repository.upenn.edu/asc_papers/43

Lombardi, M., Croce, L., Claes, C., Valdevelde, S. y Schalock, R. L. (2016). Factors predicting quality of life for people with intellectual disability: Results from the ANFFAS study in Italy. Journal of Intellectual of Developmental Disability, 41(4), 338-347. https://doi.org/10.3109/13668250.2016.1223281

Merino, C. y Livia, J. (2009). Intervalos de confianza asimétricos para el índice de validez de contenido: Un programa Visual Basic para la V de Aiken. Anales de psicología, 25(1), 169-171.

Merrells, J., Buchanan, A. y Waters, R. (2017). The experience of social inclusion for people with intellectual disability within community recreational programs: A systematic review. Journal of Intellectual \& Developmental Disability. Avance de publicación en línea. http://dx.doi.org/10.3109/13668250.2017.1283684

Montero, I. y León, O. (2007). A guide for naming research studies in Psychology. International Journal of Clinical and Health Psycho$\log y, 7(3), 847-862$.

Muñiz, J., Elosua, P. y Hambleton, R. (2013). Directrices para la traducción y adaptación de los tests: segunda edición. Psicothema, 25(2), 151-157.

Palomba, R. (2002). Calidad de vida: conceptos y medidas. Recuperado de http://www.cepal.org/celade/agenda/2/10592/envejecimiento rp1_ppt.pdf 
Pascual-García, D, Garrido-Fernández, M. y Antequera-Jurado, R. (2014). Autodeterminación y calidad de vida: un programa para la mejora de personas adultas con discapacidad intelectual. Psicología Educativa, 20(1), 33-38. https://doi.org/10.1016/j. pse.2014.05.004

Rodríguez, Y. \& Heredia, J. J. (2013). Confiabilidad ínter-observador del método de evaluación de riesgo individual. Hacia la promoción de la Salud, 18(1), 41-56.

Seligman, M., Ernstb R., Gillhamc, J., Reivicha K. y Linkinsd M. (2009) Positive education: positive psychology and classroom interventions. Review of Education, 35(3), 293-311. https://doi. org/10.1080/03054980902934563

Tamarit, J. (2005). Hacia un Sistema de Evaluación de la Calidad en FEAPS. Psychosocial Intervention, 14(3), 295-308.

Tasso, M. J., Luckasson, R. y Schalock, R. L. (2016). The relation between intellectual functioning and adaptive behavior in the diagnosis of intellectual disability. Intellectual Develo mental Disabilities, 54, 381-390. https://doi.org/10.1352/1934-955654.6.381

Tenorio, M., Arroyo R., Bunster J. y Rosas R. (2013). Identificación de la discapacidad intelectual: ¿Qué nos falta para alcanzar el estándar internacional? Notas para Educación, 14. Recuperado de www.ceppe.cl/images/stories/recursos/notas/nota-14-2.pdf

Thompson, J. R., Bradley, V. J., Buntinx, W. H. E., Schalock, R. L., Shogren, K. A., Snell, M. E., Yeager, M. H. (2009). Conceptualizing supports and the support needs of people with intellectual disability. Intellectual and Developmental Disabilities, 47(2), 135-146. https://doi.org/10.1352/1934-9556-47.2.135

Urrutia, M., Barrio, S., Gutiérrez, M. y Mayorga, M. (2014). Métodos óptimos para determinar validez de contenido. Revista Cubana de Educación Médica Superior, 28(3), 547-558.

Vargas, L. (1994). Sobre el concepto de percepción. ALTERIDADES, 4(8), 47-53. Recuperado de http://biblioteca.ues.edu.sv/revistas/ 10800277-4.pdf. 
Verdugo, M. (2001). Autodeterminación y calidad de vida de las personas con discapacidad. Propuestas de actuación. Catedrático de Psicología de la Discapacidad. INICO. Recuperado de http:// www.diversidadinclusiva.com/wp-content/uploads/2016/02/ Autodeterminacion-y-calidad-de-vida-en-las-personas-condiscapacidad-Verdugo-articulo.pdf

Verdugo, M., Gómez, L. y Arias, B. (2007). La Escala Integral de Calidad de Vida: desarrollo y estudio preliminar de sus propiedades psicométricas. Revista Española sobre Discapacidad intelectual, 38(4), 37-56.

Verdugo, M., Gómez, L., Arias, B. y Schalock, R. (2009). Escala Integral de Calidad de Vida. Madrid, España: CEPE.

Verdugo, M., Sainz F., Gómez, L. y Gómez, S. (2009). Bases para el desarrollo de un modelo de evaluación para personas con discapacidad intelectual adultas que viven en servicios residenciales. INICO: Salamanca.

Wehmeyer, M. L., Buntinx, W. H., Lachapelle y Luckasson, R. A., Schalock, R. L., Verdugo, M. A. Yeager, M. H. (2008). El constructo de discapacidad intelectual y su relación con el funcionamiento humano. Revista Española sobre Discapacidad Intelectual, 39(3), 5-18.

Recibido: 1 de julio, 2016 Revisado: 15 de febrero, 2016 Aceptado: 27 de febrero, 2017 